\title{
Advances in stereotactic body radiation therapy for early stage non-small cell lung cancer
}

\author{
Andrea M. Brown ${ }^{1}$, Raymond H. Mak ${ }^{2}$, Benjamin H. Kann ${ }^{2}$ \\ ${ }^{1}$ Columbia University, Vagelos College of Physicians and Surgeons, New York, NY, USA; ${ }^{2}$ Dana-Farber Cancer Institute/Brigham and Women's \\ Hospital, Harvard Medical School, Department of Radiation Oncology, Boston, MA, USA \\ Contributions: (I) Conception and design: All authors; (II) Administrative support: None; (III) Provision of study materials or patients: None; (IV) \\ Collection and assembly of data: R Mak, A Brown; (V) Data analysis and interpretation: B Kann, R Mak; (VI) Manuscript writing: All authors; (VII) \\ Final approval of manuscript: All authors. \\ Correspondence to: Benjamin H. Kann. Dana-Farber Cancer Institute, Department of Radiation Oncology, Boston, MA 02215, USA. \\ Email: Benjamin_Kann@DFCI.HARVARD.EDU.
}

\begin{abstract}
Non-small cell lung cancer (NSCLC) is the leading cause of cancer-related mortality in the United States, but as lung cancer screening expands to include younger patients with shorter smoking histories, an increasing number of cases are detected at early, more treatable stages. Stereotactic body radiation therapy (SBRT) has emerged as an effective, non-invasive treatment option for patients with medically inoperable NSCLC. Over the past decade, several retrospective and prospective studies have shown SBRT to achieve superior local control and overall survival compared to conventionally fractionated radiation therapy (CFRT). Advances in tumor targeting and radiation delivery have reduced treatment times while delivering higher doses of radiation to the tumor. SBRT is generally well-tolerated and the risk of grade 3 or greater toxicity is low with dose constraints in place to monitor and limit radiation delivered to surrounding normal tissues. Approaches to dose and fractionation vary depending on the tumor location relative to critical mediastinal structures, as toxicity profiles differ greatly among peripheral, central, and ultra-central tumors. While surgery remains the standard of care for operable patients and tumors, clinical trials are ongoing to compare SBRT with lobar and sub-lobar resection. Further studies combining SBRT with anti-PD-L1 and anti-PD-1 immunotherapy agents and radiosensitizing agents are in progress.
\end{abstract}

Keywords: Non-small cell lung cancer (NSCLC); stereotactic body radiation therapy (SBRT); stereotactic ablative body radiation (SABR)

Received: 28 October 2020; Accepted: 06 May 2021; Published: 25 November 2022.

doi: $10.21037 /$ ccts-21-16

View this article at: http://dx.doi.org/10.21037/ccts-21-16

\section{Introduction}

\section{Approach to radiotherapy for stage I NSCLC}

Lung cancer is the leading cause of cancer-related mortality in the United States, and over $80 \%$ of cases are classified as non-small cell lung cancer (NSCLC) (1). Though the majority of patients are diagnosed at more advanced stages with poor prognoses, increasing adoption of lung cancer screening has led to increased detection of stage I NSCLC which has more favorable outcomes (1-3). Historically, patients with stage I NSCLC treated with radiotherapy had poor outcomes, and radiotherapy was reserved for patients who were medically inoperable. Over the past two decades, groundbreaking technological advances in radiation therapy planning and delivery have led to substantially improved disease control in medically inoperable patients and has led to ongoing investigation of these newer techniques as an alternative treatment for borderline-operable and operable patients (3).

Conventionally fractionated radiation therapy (CFRT) is based upon the principle of the "Four Rs" of radiobiology: repair of tissues with sublethal damage, repopulation of cells 
between fractions, redistribution of cells into radiosensitive phases of the cell cycle, and reoxygenation of hypoxic cells to make them more radiosensitive (4). Stereotactic body radiation therapy (SBRT), which is also called stereotactic ablative body radiation (SABR), is fundamentally different from CFRT, as it uses much higher, potentially ablative doses of radiation with a reduced overall treatment time intended to limit the ability of tumors to regrow during treatment (5). While CFRT delivers treatment over the course of 6-7 weeks with daily low-dose fractions, SBRT delivers a biologically more potent dose by utilizing, generally, 1-8 days of high-dose treatments (5-7). The ability to deliver such intense daily treatments safely is owed to transformative advances in radiotherapy beam targeting, patient setup precision, and tumor motion management and tracking. These advances allow for high dose to be deposited to the tumor, with steep and rapid dose falloff to spare surrounding normal tissues (4,5). Accelerated hypofractionated radiation therapy has emerged as an intermediate option between CFRT and SBRT; it is able to deliver higher doses of radiation per fraction compared to CFRT but is suggested to be better tolerated than SBRT (8). This review will focus on SBRT which has become the standard of care for treating medically inoperable small lung nodules in light of clinical trials over the past decade showing the technique's safety and efficacy.

\section{SBRT for stage I NSCLC}

\section{Overview of SBRT techniques}

SBRT is a method of delivering high dose, highly conformal radiation to small volume extracranial sites in a small number of fractions. Approved for use by the Food and Drug Administration (FDA) in the early 2000s, the technique was originally modeled after intracranial stereotactic radiosurgery which by 1984 had accurately employed ablative doses of radiation to brain tumors by fixing the skull with a metal head frame and localizing the target with a "stereotactic" 3D coordinate system (9-11). Safe delivery of high dose-perfraction treatments to extracranial sites such as the thorax is uniquely challenging due to intra-fraction tumor motion during the respiratory cycle in addition to inter-fraction motion during daily set up. SBRT requires reproducible, rigid immobilization of the patient using an external body frame and body-contouring molds combined with real-time imaging which facilitates position adjustments for precise tumor realignment between daily fractions. Intra-fraction alignment is optimized using respiratory gating techniques, to track chest wall motion as a surrogate for internal motion during respiration, and 4D computed tomography, to construct a treatment target volume that accounts for tumor position throughout all phases of the respiratory cycle (12). With accurate and precise tumor localization, SBRT can deliver a 5- to 10-fold higher dose per fraction compared to CFRT, equating to 2-fold higher total effective dose, which increases the likelihood of tumor ablation. Furthermore, intensification of the radiation dose produces dose heterogeneity at the tumor center which further enhances tumoricidal effects while simultaneously producing a sharp gradient in dose delivered beyond the tumor (9). In the United States, SBRT is typically defined as high dose, highly conform RT delivered in 1 to 5 fractions over the course of 1 to 2 weeks, while in the rest of the world, the definition is not limited to $\leq 5$ fractions and may be delivered in up to 8-10 fractions over the course of 2 weeks (7). A timeline of advances in SBRT development is shown in Figure 1.

\section{Early experiences and longer-term outcomes with peripheral tumors}

The first phase I trial to characterize SBRT dose with acceptable toxicity was conducted by Timmerman et al. in 2003 at Indiana University (13). The study enrolled 37 patients who received 60 Gy delivered in 3 fractions, with only 2 developing grade 3 toxicity. Shortly after, the Indiana Phase II series reported the results of 70 patients with stage I NSCLC deemed medically inoperable due to FEV1 $<40 \%$ predicted, DLCO $<40 \%$ predicted, or other serious medical comorbidities precluding surgery treated with $60 \mathrm{~Gy}$ in 3 fractions (14). At a median follow-up of 50 months the rate of local control was $88 \%$. Acute grade 3-4 toxicity requiring hospitalization; characterized by decreased pulmonary function, pneumonia, pleural effusion, or skin irritation; was reported in $8(11 \%)$ patients. Death due to grade 5 toxicity; characterized by pneumonia, hemoptysis, or respiratory failure; was reported in $6(8.6 \%)$ patients. Further analysis showed that central tumors, located $<2 \mathrm{~cm}$ from the tracheobronchial tree, had higher risk (27\%) of grade 3-5 toxicity than more peripheral tumors (10\%) (15).

With this pattern of hilar and peri-central high-grade toxicity established, the multi-institutional Radiation Therapy Oncology Group (RTOG) began a phase II trial (RTOG 0236) enrolling 55 patients with medically inoperable early stage NSCLC treated per the Indiana protocol with 54 Gy delivered in 3 fractions (16). Eligible patients had tumors 


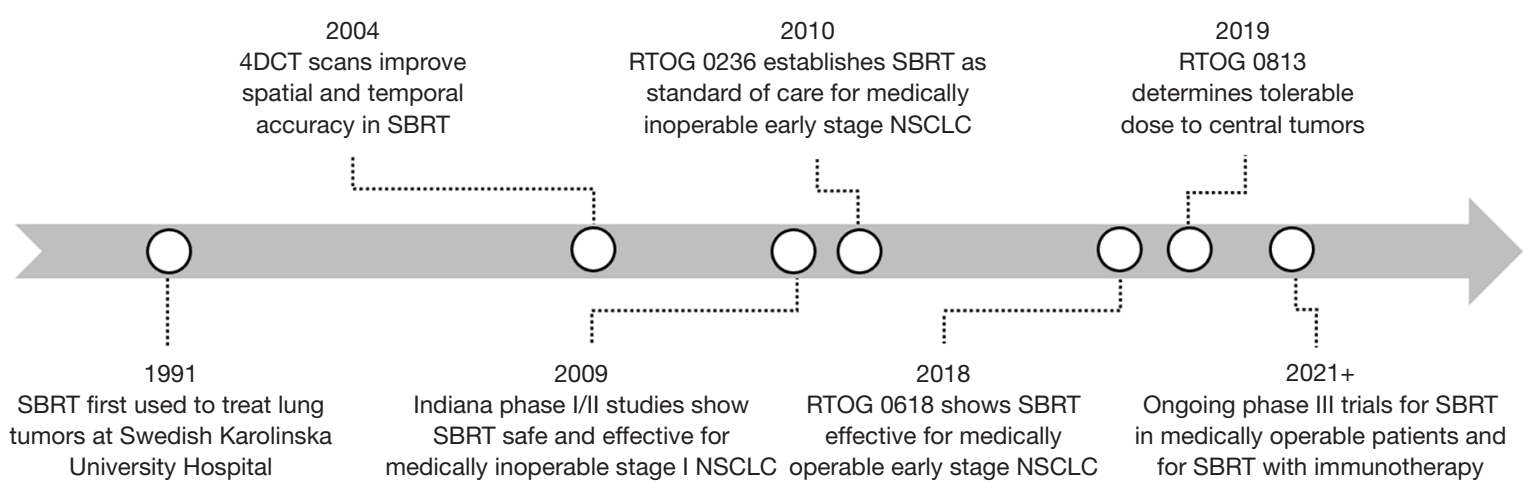

Figure 1 Timeline of advances in SBRT for NSCLC. 4DCT, four-dimensional computed tomography; RTOG, Radiation Therapy Oncology Group; SBRT, stereotactic body radiation therapy; NSCLC, non-small cell lung cancer.

$<5 \mathrm{~cm}$ in size located $>2 \mathrm{~cm}$ away from the tracheo-bronchial tree. With a median follow-up of 34 months, initial results of the study reported 3-year primary tumor control of 98\%, lobar control of $90 \%$, and overall survival of $56 \%$. In 2014 , a 5 -year update was published with a median follow-up of 48 months, reporting primary tumor control of $92.7 \%$, lobar control of $80 \%$ and overall survival of $40 \%$. Grade 3-4 toxicity was reported in $28 \%$ of patients. Although local recurrences occurred with longer term follow up, outcomes of this study appeared superior to conventional radiation therapy (coupled with a favorable safety profile, toxicity risk, and administration), and thus SBRT was established as the standard of care in the United States for most patients with medically inoperable NSCLC (7).

\section{Historical outcomes with conventional radiation therapy}

The early phase II trials determining efficacy of SBRT for early stage NSCLC achieved results superior to historical data of patients treated with CFRT (4). The 5 -year outcomes for patients treated with CFRT showed local tumor control ranging from $30-50 \%$ and overall survival of $10-15 \%$ compared to local tumor control of $90 \%$ and overall survival of $30-40 \%$ for patients treated with SBRT. These comparisons, however, were made against results achieved by the outdated technology of the two-dimensional radiation therapy era. Modern CFRT is performed using three-dimensional CT-based planning with more conformal radiation therapy techniques and image-guided treatment delivery that allow for more conformal treatment and reduced exposure of radiation dose to critical surrounding organs such as the lungs and heart $(4,6)$.

\section{Prospective data comparing SBRT versus CFRT}

No trials had directly compared SBRT to modern CFRT techniques until the SPACE trial; it was a Scandinavian, multi-center, randomized phase II trial that took place from 2007 to 2011 (17). The study enrolled 102 patients with medically inoperable stage I NSCLC and was designed to compare SBRT delivered to $66 \mathrm{~Gy}$ in 3 fractions and 3D conformal radiation therapy (3DCRT) delivered to $70 \mathrm{~Gy}$ in 35 fractions with a primary endpoint of progressionfree survival. After a median follow-up of 37 months, the trial reported a 3 -year progression-free survival of $42 \%$ in both treatment arms and a 3-year overall survival of $54 \%$ in the SBRT arm compared to $59 \%$ in the CFRT arm. Both treatment groups achieved a local control rate of $86 \%$. Patients in the SBRT arm reported lower levels of pneumonitis presenting as cough and dyspnea. Because outcomes between treatment arms were not substantially different, the question is raised whether improvements in local control associated with early SBRT trials are due to dose escalation or improved image-guidance and staging.

The CHISEL trial, a multi-center randomized phase III trial designed to compare SBRT and 3DCRT, enrolled 101 patients from 2009 to 2015 with a primary endpoint powered to detect a predicted 2-year local failure of $10 \%$ in the SBRT arm and $30 \%$ in the CFRT arm (18). After a median follow-up of 2.6 years in the SBRT arm and 2.1 years in the CFRT arm, freedom from local failure was significantly improved for SBRT (HR: 0.32; 95\% CI: $0.13-0.77 ; \mathrm{P}<0.008$ ), with 2 -year rates of local control of $86 \%$ vs. $69 \%$ for CFRT, and overall survival $77 \%$ vs. $59 \%$ for CFRT. Grade 3-4 toxicity had greater incidence in the SBRT group (12.1\%) compared to the CFRT group (5.7\%). Notable aspects of the CHISEL trial included prospective 
on-site training, end-to-end testing, and credentialing in 4D-CT planning and delivery techniques (19). This data has largely validated the widespread adoption of SBRT as the standard of care over CFRT for medically inoperable, early stage NSCLC (20).

\section{Optimal fractionation and dose}

RTOG 0236 established 54 Gy in 3 fractions as a prospectively tested approach to dose scheduling, however, several other regimens have emerged ranging from 34 Gy delivered in a single fraction to 50 Gy delivered in 10 fractions. A retrospective review of 676 patients with both operable and medically inoperable early stage NSCLC treated with SBRT at VU University Medical Center (VUMC) in Amsterdam from 2003 to 2011 evaluated a risk-adapted approach to fractionation (21). By tailoring SBRT fractionation schedules so that patients at high risk for toxicity to normal organs received lower dose per fraction, the authors aimed to limit dose-dependent pulmonary, cardiac, and esophageal toxicity. Patients with peripheral T1 tumors were treated with 54-60 Gy in 3 fractions, patients with T2 tumors or T1 tumors with broad chest wall contact were treated with 55-60 Gy in 5 fractions, and patients with tumors adjacent to the heart, hilum, or mediastinum were treated with $60 \mathrm{~Gy}$ in 8 fractions. Overall survival at 5 years was $30 \%$ and local control was $89.5 \%$, with only $3 \%$ of patients reporting grade $3+$ pneumonitis and $12 \%$ reporting chest wall pain.

The VUMC series demonstrates excellent local control and low toxicity with a risk-adapted approach, and several retrospective series have shown that high local control is associated with increasing biologically effective dose (BED), with a minimum goal threshold of at least 100-105 Gy (22-24). The BED is a measure of the true biological dose delivered to a particular tissue and varies based on the fractionation schedule and the tissue type being irradiated. For example, patients receiving 60 Gy delivered in 3 fractions receive a BED of $180 \mathrm{~Gy}$, while the same dose delivered in 8 fractions results in a BED of 105 Gy. RTOG 0915 is a phase II trial that compared SBRT regimens of 34 Gy in 1 fraction (BED =150) and 48 Gy in 4 fractions (BED $=106)$; it found similar levels of primary tumor control in both groups at 1 and 5 years (25). Grade 3-4 toxicity was lower in the group receiving $34 \mathrm{~Gy}$ in 1 fraction $(2.6 \%)$ than in the group receiving 48 Gy in 4 fractions $(11 \%)$.

Analysis of the U.S. National Cancer Database shows that 54-60 Gy in 3 fractions (BED $=151-180 \mathrm{~Gy}$ ) was the most common SBRT fractionation schedule from 2004 to 2011, but currently there is increasing utilization of 50-60 Gy in 4-5 fraction (BED =100-132 Gy), which may reduce the risk of severe side effects, but also may result in decreased tumor control (26).

\section{Central tumors}

Given the higher rates of grade 3-5 toxicity shown in the Indiana Phase II Series, 3-fraction SBRT is typically not recommended for patients with central tumors outside of clinical trials (15). These early reports created the artificial construct of the "no-fly zone" for tumors located $<2 \mathrm{~cm}$ from the proximal bronchial tree (PBT). New retrospective and prospective studies, however, have demonstrated improved toxicity rates with more fractionated regimens. A systematic review of 20 studies consisting of 563 central tumors treated with SBRT from 2000 to 2012 contends that schedules of 50 Gy in 5 fractions, 54 Gy in 6 fractions, 56 Gy in 7 fractions, and 60 Gy in 8 fractions are optimized to achieve adequate local control while minimizing the risk of treatment related mortality (27). Additionally, this review notes that most instances of grade 5 toxicity occur in patients with endobronchial tumors or in patients receiving re-irradiation of mediastinal lymph nodes. It remains important to view the risks associated with SBRT to central tumors as a continuum due to the varying definitions of a central tumor. A retrospective review by Haseltine et al. reported grade 5 toxicity in $22 \%$ of patients with "ultracentral" tumors touching the PBT (28). Represented in Figure 2, tumors $<2 \mathrm{~cm}$ from the PBT resulted in no grade 5 toxicity, though grade 3 toxicity was reported in $30 \%$ of patients with tumors within $1 \mathrm{~cm}$ of the PBT and $7 \%$ of patients with tumors 1-2 $\mathrm{cm}$ away from the PBT. Caution must be taken when treating tumors located directly adjacent to or touching airways, and safety has not yet been determined for tumors abutting the esophagus.

RTOG 0813 is a phase I/II trial that aimed to determine the maximum dose tolerated in 5 fractions for patients with central tumors (29). The study enrolled 100 medically inoperable patients with tumors located within or touching the PBT or with the radiation field touching the mediastinal or pericardial pleura. All patients were scheduled to receive 5 fractions beginning at a dose level of 10 Gy and escalating by 0.5 Gy per fraction to a maximum of 12 Gy if tolerated. The phase I analysis reported $33 \%$ of patients reaching the maximum dose, $7.2 \%$ of patients with doselimiting toxicities, and $4 \%$ of patients with treatment- 


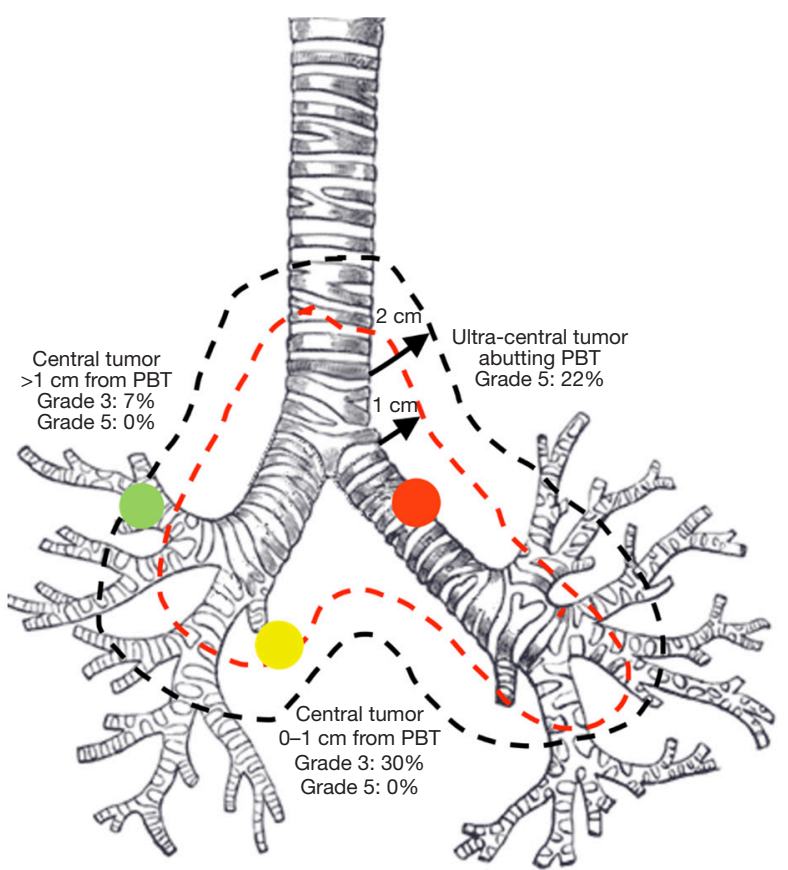

Figure 2 Grade 3+ toxicity risk according to tumor location relative to the PBT among 108 patients with NSCLC receiving SBRT with biologically effective dose $>85$ Gy (28). Central tumors, located within $2 \mathrm{~cm}$ but not touching the PBT, resulted in no grade 5 toxicity. Among central tumors, there was increased grade 3 toxicity observed as tumors approached the PBT. Grade 5 toxicity was reported in $22 \%$ of cases of ultra-central tumors located in direct contact with the PBT. PBT, proximal bronchial tree; NSCLC, non-small cell lung cancer; SBRT, stereotactic body radiation therapy.

related mortality. In the 33 patients reaching the maximum dose level of 12 Gy $\times 5$ fractions, 2-year local control was $87.9 \%$ after a median follow-up of 29.8 months, and for the 38 patients escalated to $11.5 \mathrm{~Gy} \times 5$ fractions, 2-year local control was $89.4 \%$ after a median follow-up of 33 months. The results of the phase II trial further examining treatment efficacy are forthcoming, however, this existing data suggests that SBRT is feasible for central tumors when dose to the bronchial tree can be minimized and with appropriate fractionation. Applicability of this data to ultra-central tumors is limited due to the inclusion of few ( $\mathrm{n}=17)$ ultra-central tumors, defined in this study as tumors with a planning tumor volume (PTV) abutting the mediastinum. Moreover, even at the same dose level, there may be substantial heterogeneity in the dose delivered to the PTV depending on constraints set by the treatment
Table 1 Common SBRT fraction schemes for early stage NSCLC

\begin{tabular}{|c|c|c|}
\hline Tumor position & Fraction schedule & BED \\
\hline \multirow[t]{4}{*}{ Low-risk peripheral } & $34 \times 1 f x=34$ Gy & 149.6 Gy \\
\hline & $30 \times 1 \mathrm{fx}=30$ Gy & 120 Gy \\
\hline & $20 \times 3 f x=60$ Gy & 180 Gy \\
\hline & $18 \times 3 \mathrm{fx}=54$ Gy & 151.2 Gy \\
\hline \multirow[t]{4}{*}{ High-risk peripheral } & $12.5 \times 4 \mathrm{fx}=50 \mathrm{~Gy}$ & 112.5 Gy \\
\hline & $12 \times 4$ fx $=48$ Gy & 105.6 Gy \\
\hline & $12 \times 5 \mathrm{fx}=60$ Gy & 132 Gy \\
\hline & $10 \times 5 f x=50$ Gy & 100 Gy \\
\hline \multirow[t]{5}{*}{ Central } & $12 \times 4 f x=48$ Gy & 105.6 Gy \\
\hline & $12 \times 5 \mathrm{fx}=60$ Gy & 132 Gy \\
\hline & $11 \times 5 f x=55$ Gy & 115.5 Gy \\
\hline & $10 \times 5 \mathrm{fx}=50$ Gy & 100 Gy \\
\hline & $7.5 \times 8 \mathrm{fx}=60 \mathrm{~Gy}$ & 105 Gy \\
\hline \multirow[t]{3}{*}{ Ultracentral } & $10 \times 5 f x=50$ Gy & 100 Gy \\
\hline & $9 \times 5 f x=45$ Gy & $85.5 \mathrm{~Gy}$ \\
\hline & $7.5 \times 8 \mathrm{fx}=60 \mathrm{~Gy}$ & 105 Gy \\
\hline
\end{tabular}

SBRT, stereotactic body radiation therapy; NSCLC, non-small cell lung cancer; BED, biologically effective dose; High risk peripheral tumors include those that are larger and/or in close proximity to the chest wall or other critical normal structures (e.g., brachial plexus).

plan, making it difficult to draw conclusions about the safety of the actual dose delivered to the target (30). As the classification continues to evolve towards a formal distinction between central tumors and ultra-central tumors, ASTRO currently recommends reducing toxicity risk by using 4-5 fraction regimens for central tumors and avoiding SBRT altogether for ultra-central tumors (31). Table 1 lists commonly utilized fractionation schedules according to the tumor position within the lung relative to the PBT.

\section{SBRT versus accelerated bypofractionated radiation therapy (AH-RT)}

AH-RT was developed as a strategy to reduce local recurrence rates by intensifying dose and shortening overall treatment time while also providing a, perhaps, safer side effect profile than SBRT for patients with large or central tumors (8). Regimens of 4 Gy $\times 12-15$ fractions allows AH$\mathrm{RT}$ to be better tolerated by patients with larger and more 
central tumors, however, this comes at the expense of a reduced BED, and subsequently, reduced local control (32).

Chiang et al. performed a propensity score-matched analysis comparing 119 patients treated with AH-RT delivered in 4 Gy $\times 12-15$ fractions from 1997 to 2007 and 192 patients treated with SBRT delivered in 12-12.5 Gy $\times$ 4 fractions for peripheral tumors and 10 Gy $\times 5$ fractions for central tumors from 2008 to 2012 (33). The 3-year overall survival and local control rates for the AH-RT vs. SBRT groups were $49.5 \%$ vs. $72.4 \%$ and $71.9 \%$ vs. $89.3 \%$, respectively. The study did not compare toxicity profiles. Though these results for AH-RT are unimpressive, it is important to acknowledge that the two cohorts were treated during different eras of staging and radiotherapy capabilities and that the study was retrospective and therefore subject to selection bias. Currently, the Canadian LUSTRE phase III trial is underway; this study is directly comparing SBRT and AH-RT utilizing modern radiotherapy planning and delivery technologies (34). Currently, AH-RT remains an effective, if not ideal, option for patients with high-risk tumors that are not suitable for SBRT.

\section{SBRT versus surgery}

SBRT has traditionally been limited to patients deemed unable to tolerate surgery, however, the favorable results reported in inoperable patients has led to interest in exploring SBRT for medically operable patients who prefer a noninvasive treatment (35). Potential benefits of SBRT, when compared to lobectomy, include a noninvasive, outpatient approach to treatment, with reduced short-term post-treatment mortality and effect on pulmonary reserve. On the other hand, potential disadvantages, include the lack of management of the entire lung lobe and nodal sampling, which may increase risk of locoregional failure, incomplete pathologic tissue evaluation, and late radiation side effects.

The phase II study RTOG 0618 enrolled 33 patients with medically operable, peripheral early stage NSCLC prescribed 54 Gy in 3 fractions (36). At a median followup of 48 months, 4-year overall survival and local control were $56 \%$ and $96 \%$, respectively. Grade 3 toxicity was reported in $2(8 \%)$ patients, and no grade 4 or 5 toxicity was reported. One patient underwent salvage lobectomy for local recurrence 1.2 years after SBRT, which was complicated by grade 4 cardiac arrhythmia. Although this data was impressive, subsequent randomized trials comparing lobectomy to SBRT (ROSEL, STARS) and sub-lobar resection to SBRT (ACOSOG Z4099) closed early due to poor accrual caused by patient and provider hesitancy to enroll $(37,38)$. A pooled analysis of patients from ROSEL and STARS examined 58 medically operable patients treated with SBRT or lobectomy with overall survival as the primary endpoint (37). For the 27 patients receiving surgery, median follow-up of 35.4 months was achieved with 3 -year overall survival of $79 \%$, recurrencefree survival of $80 \%$, and local control of $100 \%$. For the 31 patients receiving SBRT, a median follow-up of 40.2 months was achieved with 3 -year overall survival of $95 \%$, recurrence-free survival of $86 \%$, and local control of $96 \%$. Grade 3-4 toxicity occurred in 12 (44\%) of patients receiving surgery and $3(10 \%)$ of patients in the SBRT arm. One death occurred due to surgical complications and none due to SBRT. The follow-up and sample size of this pooled analysis are too small to make definitive conclusions, but these findings suggest that data from larger trials are needed.

Trials comparing sub-lobar resection and SBRT are ongoing. STABLE-MATES (NCT02468024), a phase III study of high-risk surgical candidates randomized to sublobar resection $v s$. SBRT before consultation, is scheduled to be completed in 2024. VALOR (NCT02984761) is a phase III randomized trial of anatomic resection $v s$. SBRT with an expected completion date in 2027. Currently the American Society for Radiation Oncology (ASTRO) recommends that radiation oncologists be prepared to engage in shared decision making with patients when discussing SBRT as a potential alternative to surgery for high-risk operable candidates with stage I NSCLC (39).

Early experiences leading to the incorporation of SBRT into the standard of care for medically inoperable NSCLC understandably have been met with some caution. Pathologic confirmation of disease is important for the appropriately diagnosing and staging cancers, and since many patients undergoing SBRT are unable to have tissue sampled, some theorize that the possible inclusion of hamartomas, granulomas, and other non-cancerous lesions could overestimate efficacy in treating NSCLC with SBRT $(40,41)$. Additionally, there has been noted variability in the criteria used to define patient operability and local or regional failures (41). In order to minimize the risk of bias, many ongoing clinical trials are designed with close supervision of human research protection monitoring, and in the case of the SABRTooth trial (NCT02629458) comparing SBRT with surgery, potentially eligible patients are enrolled by a pulmonologist and only meet the specialist 
relevant to their treatment arm after randomization (35).

\section{Toxicity}

The toxicity profile of SBRT differs from that of conventional thoracic radiation therapy, nevertheless, the largest series suggest that risk of grade 3-4 toxicity remains low $(14,31,42)$. Maintaining this low incidence of severe toxicity is heavily dependent on selecting the appropriate fractionation regimen using a risk-adapted approach as outlined above, and by the radiation oncologists carefully understanding and respecting currently published dosimetric constraints to critical organs at risk such as the mediastinal structures for central tumors and lung and chest wall for peripheral tumors $(15,29,43,44)$.

The most common adverse effect is late onset chest wall pain which is reported in $10-21 \%$ of patients and has a median onset of 6-9 months after completing treatment. Rib fractures may occur in $3-21 \%$ of patients, though they are often asymptomatic. A study from the University of Toronto performed a multivariate analysis of 289 NSCLC tumors treated with 48-64 Gy in 4 fractions and found that tumor location adjacent to the chest was a significant predictor for rib fracture but found no predictors for chest wall pain without an underlying rib fracture (45).

Other uncommon toxicities include radiation pneumonitis, esophagitis, and brachial plexopathy. Pulmonary toxicity secondary to radiation pneumonitis is of greatest concern since most NSCLC patients have poor baseline lung function (46). Radiation pneumonitis classically consists of dyspnea on exertion, non-productive cough, hypoxemia, and/or low-grade fevers with associated imaging findings in the irradiated field. RTOG 0236, the phase II trial that established the efficacy of 54 Gy in 3 fractions, enrolled a cohort with a mean baseline FEV1 of $60.8 \%$ predicted and DLCO $60.7 \%$ predicted $(15,47)$. At 2-year follow-up, these values had decreased by $5.8 \%$ and $6.3 \%$, respectively. Grade 3-4 pulmonary toxicity was reported in $16 \%$ of patients with no correlation found between baseline PFT parameters and cough, dyspnea, hypoxia, pneumonitis, or PFT decrease after treatment with SBRT. Long term effect on pulmonary function requires further study.

Radiation pneumonitis presents an additional challenge in differentiation evolving fibrosis from recurrence. Acute lung parenchyma changes typically begin 3-6 months following SBRT, with radiation fibrosis beginning at 6-9 months and stabilizing by $1-2$ years (48). Because SBRT uses complex dose distributions, the pattern of fibrosis can appear dense, non-linear, and mass-like on follow up radiographic imaging whereas conventional radiation therapy produces fibrosis with linear borders. PET-CT may be helpful to resolve equivocal cases, but low-level uptake due to inflammation may remain present for several years, necessitating a biopsy for definitive diagnosis $(49,50)$.

\section{Future directions}

With numerous clinical trials in progress, the utilization of SBRT for early stage NSCLC continues to grow. Table 2 summarizes outcomes achieved by SBRT in selected prospective studies previously discussed. Lung cancer is a disease of the elderly and as the population ages, patients may present more often with comorbidities that preclude lobectomy. The results of trials such as VALOR and STABLE-MATES comparing SBRT with sublobar resection have the potential to empirically expand indications for SBRT to these high-risk patients. In the United Kingdom, SABRTooth is a phase III trial aiming to compare SBRT to surgical resection while avoiding the pitfalls of specialty bias in patient enrollment, and in China, POSTLIV (NCT01753414) is a phase III trial aiming to compare locoregional control in SBRT versus radical surgical resection.

Recently, the United States Preventative Services and Task Force (USPSTF) updated its recommendation for annual screening for lung cancer with low-dose CT to include adults between ages 50-80 who have a 20 pack-year or greater smoking history and currently smoke or have quit within that last 15 years (51). The guidelines continue to recommend, however, that patients unwilling or unable to undergo curative surgery due to health problems that may substantially limit life expectancy discontinue screening (52). This stipulation does not take into account the advances in SBRT as a non-invasive approach for treating patients ineligible for surgery. As the USPSTF recognizes the improved outcomes for patients diagnosed with early-stage lung cancer, in the future it is likely necessary to consider expanding access to screening to patients ineligible for surgery since SBRT is an effective alternative (53).

The past decade has also seen the rapid advancement of immunotherapy as a fourth pillar of oncologic care. Radiation may work synergistically with immunotherapy by increasing MHC class I expression on tumor cells thereby reactivating the immune system within the tumor microenvironment $(54,55)$. Now the standard of care 
Table 2 Summary of outcomes and reported toxicity in selected prospective studies of SBRT for early stage NSCLC

\begin{tabular}{|c|c|c|c|c|c|}
\hline Trial (reference) & Tumor Location [n] & Fractionation [n] & LC & OS & Grade 3+ Toxicity [n] \\
\hline RTOG 0236 (16) & Peripheral [55] & 54 Gy/3 fx [55] & $\begin{array}{l}90 \% \text { at } 3 \text { years } \\
80 \% \text { at } 5 \text { years }\end{array}$ & $\begin{array}{l}56 \% \text { at } 3 \text { years } \\
40 \% \text { at } 5 \text { years }\end{array}$ & $\begin{array}{l}16 \%[9] \text { respiratory } \\
5 \%[3] \text { hematologic } \\
5 \% \text { [3] musculoskeletal } \\
4 \% \text { [2] dermatologic } \\
4 \% \text { [2] infection } \\
2 \% \text { [1] gastrointestinal } \\
2 \% \text { [1] metabolic } \\
2 \% \text { [1] neurologic } \\
2 \% \text { [1] constitutional }\end{array}$ \\
\hline RTOG 0915 (25) & Peripheral [84] & $\begin{array}{l}48 \text { Gy/4 fx [45] } \\
34 \text { Gy/1 fx [39] }\end{array}$ & Not reported & $61-78 \%$ at 2 years & $\begin{array}{l}8 \% \text { [7] decreased PFTs } \\
2 \% \text { [2] pneumonitis } \\
1 \% \text { [1] respiratory failure }\end{array}$ \\
\hline ROSEL/STARS (37) & $\begin{array}{l}\text { Peripheral [27] } \\
\text { Central [4] }\end{array}$ & $\begin{array}{l}60 \mathrm{~Gy} / 5 \mathrm{fx}[5] \\
54 \mathrm{~Gy} / 3 \mathrm{fx}[22] \\
50 \mathrm{~Gy} / 4 \mathrm{fx}[4]\end{array}$ & $96 \%$ at 3 years & $95 \%$ at 3 years & $\begin{array}{l}10 \% \text { [3] chest wall pain } \\
6 \% \text { [2] dyspnea } \\
3 \% \text { [1] rib fracture }\end{array}$ \\
\hline SPACE (17) & Peripheral [48] & 66 Gy/3 fx [48] & $86 \%$ at 3 years & $54 \%$ at 3 years & $\begin{array}{l}10 \%[5] \text { dyspnea } \\
2 \% \text { [1] cough } \\
2 \% \text { [1] skin reaction }\end{array}$ \\
\hline CHISEL (18) & Peripheral [63] & $\begin{array}{l}54 \text { Gy/3 fx [8] } \\
48 \text { Gy/4 fx [55] }\end{array}$ & $86 \%$ at 2 years & $77 \%$ at 2 years & $\begin{array}{l}3 \%[2] \text { dyspnea } \\
3 \%[2] \text { cough } \\
2 \%[1] \text { fatigue } \\
2 \% \text { [1] lung infection } \\
2 \% \text { [1] hypoxia } \\
2 \% \text { [1] weight loss }\end{array}$ \\
\hline
\end{tabular}

SBRT, stereotactic body radiation therapy; NSCLC, non-small cell lung cancer; LC, local control; OS, overall survival; PFTs, pulmonary function tests.

for unresectable stage III NSCLC, durvalumab, a PDL1 monoclonal antibody, is being combined with SBRT for unresected early stage NSCLC as the treatment arm of the phase III trial PACIFIC-4 (NCT03833154) (56). Similarly, additional phase II and III trials are underway evaluating SBRT alone versus SBRT in combination with other immunotherapy agents including the PD-L1 monoclonal antibody atezolizumab in the SWOG 1914 trial (NCT04214262) and the PD-1 monoclonal antibodies nivolumab, in the I-SABR trial (NCT03110978), and pembrolizumab, in the Keynote-867 trial (NCT03924869).

Finally, stereotactic magnetic resonance-guided 
adaptive radiation therapy (SMART) is an exciting, emerging technique that employs daily treatment target reoptimization to account for small variations in the treatment volume and nearby organs at risk, permitting a widened therapeutic index when treating ultra-central tumors (57). Combining radiation therapy with novel sensitizing agents, including testing SMART with radiosensitizing theranostic nanoparticles or concurrent immunotherapy with SBRT, are also being investigated as the next frontier for SBRT advancement (58).

\section{Conclusions}

The last two decades have produced a body of evidence establishing SBRT as a safe, efficacious, and non-invasive way of treating medically inoperable stage I NSCLC. SBRT has progressed at a rapid pace with impressive outcomes; however, surgical resection remains the standard of care for patients with medically operable stage I NSCLC, as data directly comparing lobectomy to SBRT are limited. There is particularly good evidence for treating peripheral tumors with additional strategies being developed to limit chest wall toxicity. Optimal treatment regimens for central tumors remains under study as further classification of these tumors according to degree of centrality and which critical structure the tumor abuts remains ongoing. In borderline resectable patients, deciding between SBRT and surgical resection requires collaboration between the patient and the multidisciplinary team. Moving forward, clinical trials comparing the best radiotherapy approaches against the best surgical approaches with standardized definitions of patient suitability for each approach will help optimize treatment efficacy while minimizing morbidity.

\section{Acknowledgments}

Funding: None.

\section{Footnote}

Provenance and Peer Review: This article was commissioned by the Guest Editors (Scott Swanson, Daniel Dolan) for the series "How to Evaluate, Diagnose and Treat Small Lung Nodules" published in Current Challenges in Thoracic Surgery. The article has undergone external peer review.

Peer Review File: Available at https://ccts.amegroups.com/ article/view/10.21037/ccts-21-16/prf
Conflicts of Interest: All authors have completed the ICMJE uniform disclosure form (available at https:// ccts.amegroups.com/article/view/10.21037/ccts-21-16/ coif). The series "How to Evaluate, Diagnose and Treat Small Lung Nodules" was commissioned by the editorial office without any funding or sponsorship. RHM reports consulting fees from AstraZeneca and ViewRay, grants from ViewRay and payment from US Attorney's Office Northern District of New York. The authors have no other conflicts of interest to declare.

Ethical Statement: The authors are accountable for all aspects of the work in ensuring that questions related to the accuracy or integrity of any part of the work are appropriately investigated and resolved.

Open Access Statement: This is an Open Access article distributed in accordance with the Creative Commons Attribution-NonCommercial-NoDerivs 4.0 International License (CC BY-NC-ND 4.0), which permits the noncommercial replication and distribution of the article with the strict proviso that no changes or edits are made and the original work is properly cited (including links to both the formal publication through the relevant DOI and the license). See: https://creativecommons.org/licenses/by-nc-nd/4.0/.

\section{References}

1. Lu T, Yang X, Huang $Y$, et al. Trends in the incidence, treatment, and survival of patients with lung cancer in the last four decades. Cancer Manag Res 2019;11:943-53.

2. Duffy SW, Field JK. Mortality Reduction with LowDose CT Screening for Lung Cancer. N Engl J Med 2020;382:572-3.

3. Sebastian NT, Xu-Welliver M, Williams TM. Stereotactic body radiation therapy (SBRT) for early stage non-small cell lung cancer (NSCLC): contemporary insights and advances. J Thorac Dis 2018;10:S2451-64.

4. Bucci MK, Bevan A, Roach M 3rd. Advances in radiation therapy: conventional to $3 \mathrm{D}$, to IMRT, to $4 \mathrm{D}$, and beyond. CA Cancer J Clin 2005;55:117-34.

5. Gaya A, Mahadevan A. Stereotactic body radiotherapy : a practical guide. London: Springer-Verlag, 2015.

6. Cai J, Chang JY, Yin FF. Principles and Practice of ImageGuided Radiation Therapy of Lung Cancer. Boca Raton, Florida: CRC Press, 2017.

7. Potters L, Kavanagh B, Galvin JM, et al. American Society for Therapeutic Radiology and Oncology (ASTRO) and 
American College of Radiology (ACR) practice guideline for the performance of stereotactic body radiation therapy. Int J Radiat Oncol Biol Phys 2010;76:326-32.

8. Soliman H, Cheung P, Yeung L, et al. Accelerated hypofractionated radiotherapy for early-stage non-smallcell lung cancer: long-term results. Int $\mathrm{J}$ Radiat Oncol Biol Phys 2011;79:459-65.

9. Nagata Y. Stereotactic body radiation therapy : principles and practices. Japan: Springer, 2015.

10. Blomgren H, Lax I, Näslund I, et al. Stereotactic high dose fraction radiation therapy of extracranial tumors using an accelerator. Clinical experience of the first thirty-one patients. Acta Oncol 1995;34:861-70.

11. Uematsu M, Shioda A, Tahara K, et al. Focal, high dose, and fractionated modified stereotactic radiation therapy for lung carcinoma patients: a preliminary experience. Cancer 1998;82:1062-70.

12. Underberg RW, Lagerwaard FJ, Cuijpers JP, et al. Four-dimensional CT scans for treatment planning in stereotactic radiotherapy for stage I lung cancer. Int J Radiat Oncol Biol Phys 2004;60:1283-90.

13. Timmerman R, Papiez L, McGarry R, et al. Extracranial stereotactic radioablation: results of a phase I study in medically inoperable stage I non-small cell lung cancer. Chest 2003;124:1946-55.

14. Fakiris AJ, McGarry RC, Yiannoutsos CT, et al. Stereotactic body radiation therapy for early-stage non-small-cell lung carcinoma: four-year results of a prospective phase II study. Int J Radiat Oncol Biol Phys 2009;75:677-82.

15. Timmerman R, McGarry R, Yiannoutsos C, et al. Excessive toxicity when treating central tumors in a phase II study of stereotactic body radiation therapy for medically inoperable early-stage lung cancer. J Clin Oncol 2006;24:4833-9.

16. Timmerman R, Paulus R, Galvin J, et al. Stereotactic body radiation therapy for inoperable early stage lung cancer. JAMA 2010;303:1070-6.

17. Nyman J, Hallqvist A, Lund JA, et al. SPACE - A randomized study of SBRT vs conventional fractionated radiotherapy in medically inoperable stage I NSCLC. Radiother Oncol 2016;121:1-8.

18. Ball D, Mai GT, Vinod S, et al. Stereotactic ablative radiotherapy versus standard radiotherapy in stage 1 nonsmall-cell lung cancer (TROG 09.02 CHISEL): a phase 3, open-label, randomised controlled trial. Lancet Oncol 2019;20:494-503.

19. Joseph N, Choudhury A. SABR versus conventional fractionation regimens in NSCLC. Lancet Oncol 2019;20:e231.

20. Kron T, Chesson B, Hardcastle N, et al. Credentialing of radiotherapy centres in Australasia for TROG 09.02 (Chisel), a Phase III clinical trial on stereotactic ablative body radiotherapy of early stage lung cancer. Br J Radiol 2018;91:20170737.

21. Senthi S, Lagerwaard FJ, Haasbeek CJ, et al. Patterns of disease recurrence after stereotactic ablative radiotherapy for early stage non-small-cell lung cancer: a retrospective analysis. Lancet Oncol 2012;13:802-9.

22. Grills IS, Hope AJ, Guckenberger M, et al. A collaborative analysis of stereotactic lung radiotherapy outcomes for early-stage non-small-cell lung cancer using daily online cone-beam computed tomography image-guided radiotherapy. J Thorac Oncol 2012;7:1382-93.

23. Onishi H, Shirato H, Nagata $Y$, et al. Hypofractionated stereotactic radiotherapy (HypoFXSRT) for stage I nonsmall cell lung cancer: updated results of 257 patients in a Japanese multi-institutional study. J Thorac Oncol 2007;2:S94-100.

24. Wulf J, Baier K, Mueller G, et al. Dose-response in stereotactic irradiation of lung tumors. Radiother Oncol 2005;77:83-7.

25. Videtic GM, Stephans KL, Woody NM, et al. 30 Gy or 34 Gy? Comparing 2 single-fraction SBRT dose schedules for stage I medically inoperable non-small cell lung cancer. Int J Radiat Oncol Biol Phys 2014;90:203-8.

26. Corso CD, Park HS, Moreno AC, et al. Stage I Lung SBRT Clinical Practice Patterns. Am J Clin Oncol 2017;40:358-61.

27. Senthi S, Haasbeek CJ, Slotman BJ, et al. Outcomes of stereotactic ablative radiotherapy for central lung tumours: a systematic review. Radiother Oncol 2013;106:276-82.

28. Haseltine JM, Rimner A, Gelblum DY, et al. Fatal complications after stereotactic body radiation therapy for central lung tumors abutting the proximal bronchial tree. Pract Radiat Oncol 2016;6:e27-33.

29. Bezjak A, Paulus R, Gaspar LE, et al. Safety and Efficacy of a Five-Fraction Stereotactic Body Radiotherapy Schedule for Centrally Located Non-Small-Cell Lung Cancer: NRG Oncology/RTOG 0813 Trial. J Clin Oncol 2019;37:1316-25.

30. Chaudhuri AA, Tang C, Binkley MS, et al. Stereotactic ablative radiotherapy (SABR) for treatment of central and ultra-central lung tumors. Lung Cancer 2015;89:50-6.

31. Thompson M, Rosenzweig KE. The evolving toxicity profile of SBRT for lung cancer. Transl Lung Cancer Res 
2019;8:48-57.

32. Cheung P, Faria S, Ahmed S, et al. Phase II study of accelerated hypofractionated three-dimensional conformal radiotherapy for stage T1-3 N0 M0 non-small cell lung cancer: NCIC CTG BR.25. J Natl Cancer Inst 2014;106:dju164.

33. Chiang A, Thibault I, Warner A, et al. A comparison between accelerated hypofractionation and stereotactic ablative radiotherapy (SABR) for early-stage non-small cell lung cancer (NSCLC): Results of a propensity scorematched analysis. Radiother Oncol 2016;118:478-84.

34. Swaminath A, Wierzbicki M, Parpia S, et al. Canadian Phase III Randomized Trial of Stereotactic Body Radiotherapy Versus Conventionally Hypofractionated Radiotherapy for Stage I, Medically Inoperable NonSmall-Cell Lung Cancer - Rationale and Protocol Design for the Ontario Clinical Oncology Group (OCOG)LUSTRE Trial. Clin Lung Cancer 2017;18:250-4.

35. Tian S, Higgins KA, Curran WJ, et al. Stereotactic body radiation therapy vs. surgery in early-stage non-small cell lung cancer: lessons learned, current recommendations, future directions. J Thorac Dis 2018;10:1201-4.

36. Timmerman RD, Paulus R, Pass HI, et al. Stereotactic Body Radiation Therapy for Operable Early-Stage Lung Cancer: Findings From the NRG Oncology RTOG 0618 Trial. JAMA Oncol 2018;4:1263-6.

37. Chang JY, Senan S, Paul MA, et al. Stereotactic ablative radiotherapy versus lobectomy for operable stage I nonsmall-cell lung cancer: a pooled analysis of two randomised trials. Lancet Oncol 2015;16:630-7.

38. Hurkmans CW, Cuijpers JP, Lagerwaard FJ, et al. Recommendations for implementing stereotactic radiotherapy in peripheral stage IA non-small cell lung cancer: report from the Quality Assurance Working Party of the randomised phase III ROSEL study. Radiat Oncol 2009; 4:1.

39. Paruch JL, Ko CY, Bilimoria KY. An opportunity to improve informed consent and shared decision making: the role of the ACS NSQIP Surgical Risk Calculator in oncology. Ann Surg Oncol 2014;21:5-7.

40. Ettinger DS, Wood DE, Aggarwal C, et al. NCCN Guidelines Insights: Non-Small Cell Lung Cancer, Version 1.2020. J Natl Compr Canc Netw 2019;17:1464-72.

41. White A, Swanson SJ. Surgery versus stereotactic ablative radiotherapy (SABR) for early-stage non-small cell lung cancer: less is not more. J Thorac Dis 2016;8:S399-405.

42. Lagerwaard FJ, Haasbeek CJ, Smit EF, et al. Outcomes of risk-adapted fractionated stereotactic radiotherapy for stage I non-small-cell lung cancer. Int J Radiat Oncol Biol Phys 2008;70:685-92.

43. Kong FS, Moiseenko V, Zhao J, et al. Organs at Risk Considerations for Thoracic Stereotactic Body Radiation Therapy: What Is Safe for Lung Parenchyma? Int J Radiat Oncol Biol Phys 2021;110:172-87.

44. Zhao J, Yorke ED, Li L, et al. Simple Factors Associated With Radiation-Induced Lung Toxicity After Stereotactic Body Radiation Therapy of the Thorax: A Pooled Analysis of 88 Studies. Int J Radiat Oncol Biol Phys 2016;95:1357-66.

45. Thibault I, Chiang A, Erler D, et al. Predictors of Chest Wall Toxicity after Lung Stereotactic Ablative Radiotherapy. Clin Oncol (R Coll Radiol) 2016;28:28-35.

46. Bishawi M, Kim B, Moore WH, et al. Pulmonary function testing after stereotactic body radiotherapy to the lung. Int J Radiat Oncol Biol Phys 2012;82:e107-10.

47. Stanic S, Paulus R, Timmerman RD, et al. No clinically significant changes in pulmonary function following stereotactic body radiation therapy for early- stage peripheral non-small cell lung cancer: an analysis of RTOG 0236. Int J Radiat Oncol Biol Phys 2014;88:1092-9.

48. Linda A, Trovo M, Bradley JD. Radiation injury of the lung after stereotactic body radiation therapy (SBRT) for lung cancer: a timeline and pattern of CT changes. Eur J Radiol 2011;79:147-54.

49. Guckenberger M, Heilman K, Wulf J, et al. Pulmonary injury and tumor response after stereotactic body radiotherapy (SBRT): results of a serial follow-up CT study. Radiother Oncol 2007;85:435-42.

50. Matsuo Y, Nagata Y, Mizowaki T, et al. Evaluation of mass-like consolidation after stereotactic body radiation therapy for lung tumors. Int J Clin Oncol 2007;12:356-62.

51. Krist AH, Davidson KW, Mangione CM, et al. Screening for Lung Cancer: US Preventive Services Task Force Recommendation Statement. JAMA 2021;325:962-70.

52. Moyer VA. Screening for lung cancer: U.S. Preventive Services Task Force recommendation statement. Ann Intern Med 2014;160:330-8.

53. Force USPST. U.S. Preventive Services Task Force Issues Draft Recommendation Statement on Screening for Lung Cancer. USPSTF Bulletin 2020.

54. Lavin Y, Kobayashi S, Leader A, et al. Innate Immune Landscape in Early Lung Adenocarcinoma by Paired Single-Cell Analyses. Cell 2017;169:750-65.e17.

55. Lin AJ, Roach M, Bradley J, et al. Combining stereotactic body radiation therapy with immunotherapy: current data and future directions. Transl Lung Cancer Res 
2019;8:107-15.

56. Antonia SJ, Villegas A, Daniel D, et al. Overall Survival with Durvalumab after Chemoradiotherapy in Stage III NSCLC. N Engl J Med 2018;379:2342-50.

57. Henke LE, Olsen JR, Contreras JA, et al. Stereotactic MRGuided Online Adaptive Radiation Therapy (SMART) for
Ultracentral Thorax Malignancies: Results of a Phase 1 Trial. Adv Radiat Oncol 2019;4:201-9.

58. Detappe A, Thomas E, Tibbitt MW, et al. Ultrasmall Silica-Based Bismuth Gadolinium Nanoparticles for Dual Magnetic Resonance-Computed Tomography Image Guided Radiation Therapy. Nano Lett 2017;17:1733-40.

doi: $10.21037 /$ ccts-21-16

Cite this article as: Brown AM, Mak RH, Kann BH. Advances in stereotactic body radiation therapy for early stage non-small cell lung cancer. Curr Chall Thorac Surg 2022;4:41. 